\title{
Vascular impact of quercetin administration in association with moderate exercise training in experimental type 1 diabetes
}

\author{
Irina Camelia Chis ${ }^{1 *}$, Mihai Socaciu ${ }^{2}$, Remus Moldovan ${ }^{1}$, Simona Clichici ${ }^{1}$ \\ 1. Department of Physiology, "Iuliu Hațieganu” University of Medicine and Pharmacy, Cluj Napoca, \\ Romania \\ 2. Ultrasonography Department, $3^{\text {rd }}$ Medical Clinic, "Iuliu Haţieganu” University of Medicine and \\ Pharmacy, Cluj Napoca, Romania
}

\begin{abstract}
Hyperglycemia and oxidative stress have a major role in the pathogenesis of diabetic vascular complications. In this study, we investigated the efficacy of combining quercetin treatment with moderate exercise training in reversing diabetes-induced oxidative stress and ultrasound modifications in rat carotid arteries. The diabetic Wistar rats were divided into sedentary groups and trained groups. The trained animals went through a regular moderate exercise by swimming (5 weeks). Some non-diabetic and diabetic rats were daily treated with quercetin (30 mg/kg, for 5 weeks). At the end of the study, the imaging evaluation required to assess the effects of diabetes on carotid arteries was performed by micro-ultrasound (MU). The diabetic rats presented atherosclerotic plaques, with an increase in the echogenicity of the carotid artery wall, carotid intima-media thickness (CIMT), and carotid wall thickness, while the diabetic trained rats treated with quercetin presented normal values of these parameters. Malondialdehyde (MDA) levels, superoxide dismutase (SOD) antioxidant enzyme activity, reduced glutathione (GSH) levels and the reduced (GSH) to oxidized (GSSG) glutathione ratio were determined in the carotid artery tissue. Diabetes caused elevated MDA levels and a decrease in SOD activity, GSH levels and GSH/GSSG ratio in the carotid artery tissue. Treating diabetic rats with quercetin combined with moderate exercise training reversed all these oxidative stress parameters. Our results show that this combination, quercetin and moderate exercise training, can be a good treatment strategy for the vascular complications of diabetes by attenuating hyperglycemia-mediated oxidative stress.
\end{abstract}

Keywords: carotid arteries, diabetes mellitus, oxidative stress, quercetin, ultrasonography

Received: 23 ${ }^{\text {rd }}$ December 2018; Accepted: 23rd May 2019; Published: $13^{\text {th }}$ June 2019

*Corresponding author: Irina Camelia Chis, Department of Physiology, 1-3, Clinicilor Street, "Iuliu Hatieganu" University of Medicine and Pharmacy, 400023, Cluj-Napoca, Romania.

E-mail: irinnaus@yahoo.com, ichis@umfcluj.ro 


\section{Introduction}

Type 1 diabetes mellitus (T1DM) is an autoimmune disorder characterised by insulin deficiency which affects resistance arteries and conduit arteries such as the carotid arteries. T1DM is associated with endothelial dysfunction and atherosclerosis $(1,2)$. The micro- and macrovascular complications of diabetes are a consequence of endothelial dysfunction and inflammation. The major causal factors involved in the pathogenesis of endothelial dysfunction in T1DM are hyperglycemia, dyslipidemia and oxidative stress (3-6). Atherosclerosis represents the principal cause of disability and death in patients with T1DM. The development of atherosclerosis and cardiovascular complications can be prevented by maintaining endothelial function.

Despite administration of appropriate treatment, in the long-term, T1DM leads in most cases to microvascular, macrovascular and neurological complications, such as cardiomyopathy, atherosclerosis, nephropathy, retinopathy and neuropathy $(1,6)$. Diabetes accelerates the formation of atherosclerotic plaques and intimal proliferation in the carotid artery by vascular smooth muscle cell proliferation, inflammatory cell infiltration, endothelial cell injury and augmented deposition of extracellular matrix (7).

Polyphenols represent a large group of bioactive plant compounds known for their many beneficial effects on the body: antioxidant, antiatherogenic, hypoglycemic, hypocholesterolemic, hypotriglyceridemic, anti-inflammatory, antimicrobial, etc. (8). The flavonoid quercetin is one of the most important natural antioxidants found in many vegetables and fruits (9). Quercetin exerts multiple biological effects - antioxidant, hypoglycemic, neuroprotective and anti-inflammatory - in T1DM $(3,10-17)$. Recent research has demonstrated the beneficial effects of exercise training on preventing diabetic vascular compli- cations by reducing oxidative/nitrosative stress and inflammation $(3,14,18-22)$.

Therefore, in the current study we evaluated the effects of combining quercetin treatment with moderate exercise training in preventing carotid artery injuries in diabetic rats through a reduction of glycemia and oxidative stress. This original research study evaluates in vivo carotid artery ultrasound changes induced by streptozotocin and prevention of vascular damage by quercetin administration combined with moderate exercise training.

\section{Material and methods}

\section{Experimental animal design}

Wistar albino rats aged 3 months (230-260 g weight) were purchased from the Experimental Animal House of the "Iuliu Hațieganu" University of Medicine and Pharmacy (Cluj-Napoca, Romania). They were maintained in a controlled environment $(12: 12 \mathrm{~h}$ light/dark cycle, at a temperature of $21-23^{\circ} \mathrm{C}$ and $50 \%-60 \%$ humidity). Standard pellet diet and water were provided $a d$ libitum. Animal experimental and welfare procedures were conducted in accordance with the Guide for the Care and Use of Laboratory Animals (The Romanian Ministry of Health) and complying with the approved animal protocols (No. 78/20.02.2014) of the Ethical Committee on Animal Welfare. Streptozotocin (STZ) $(50 \mathrm{mg} /$ $\mathrm{kg}$ body weight) (Sigma-Aldrich, Inc., UK) was administered by a single intraperitoneal (i.p.) injection, after having been dissolved in $0.1 \mathrm{~mol} / \mathrm{L}$ sodium citrate buffer ( $\mathrm{pH} 4.5)$, and the non-diabetic control rats received only sodium citrate buffer $(0.1 \mathrm{~mol} / \mathrm{L})(3,23-28)$. Subsequently, 96 $\mathrm{h}$ after the STZ injection, T1DM was confirmed by assessing fasting blood glucose (FBG) levels in blood samples collected from the tail of rats deprived of food overnight. The rats whose FBG levels were above $250 \mathrm{mg} / \mathrm{dL}$ were considered to be diabetic. Quercetin treatment and exercise 
training started 7 days after STZ administration. The FBG levels were recorded using a glucose test strip (ACCU-CHEK Go glucometer, Roche) before (initial FBG) and $96 \mathrm{~h}$ after STZ administration, 7 days after STZ administration, and at the end of the experiment (final FBG). Body weight (BW) was also measured at the start (initial BW) and at the end (final BW) of the experiment.

The experimental rats were randomly divided into control (non-diabetic) and diabetic groups $(n=10)$ : the CS group: control sedentary rats; the CE group: control trained rats; the CSQ group: control sedentary rats that received quercetin; the CEQ group: control trained rats with quercetin administration; the DS group: diabetic sedentary rats; the DE group: diabetic trained rats; the DSQ group: diabetic sedentary rats that received quercetin; the DEQ group: trained diabetic rats with quercetin administraion. Quercetin (Sigma-Aldrich, Inc., UK) was suspended in $0.5 \%$ carboxymethylcellulose (CMC) solution and then administered via intragastric gavage (30 $\mathrm{mg} / \mathrm{kg}$ body weight, once daily for 5 weeks). CMC solution was administered by gavage once daily ( 5 weeks) to the rats of groups: CS, CE, DS and DE $(0.6 \mathrm{ml} / \mathrm{rat})$.

\section{Exercise training protocol}

The physical training program consisted of moderate swimming 30 minutes/day, 5 days/week (for 5 weeks), as previously described $(13,14)$, using a modification of the protocol described by Pyun et al. (18) and Teixeira de Lemos et al. (19). The control and diabetic sedentary rats were handled daily and treated similarly to the trained rats except for the swimming training.

\section{Ultrasound protocol}

All the ultrasonographic evaluations required to assess the effects of DM on the vascular endothelium (mostly atherosclerosis, with its consequences) were performed by micro-ultrasound by a single examiner experienced in both clinical and experimental ultrasound (M.S.). High performance micro-ultrasound equipment, specifically designed for the examination of small experimental animals, was used: a VisualSonics Vevo 2100 high frequency machine equipped with an imaging station for rat immobilisation, monitoring and scanning. An 18-24 MHz linear transducer was used for the screening of atheromatous plaques in the carotid arteries, as well as for Doppler measurements. The wall thickness of the carotid arteries was measured on transverse sections using a 50-70 MHz transducer.

The rats from each group were anesthetised using an i.p. injection of ketamine $(100 \mathrm{mg} / \mathrm{kg}$ BW) and xylazine (10 mg/kg BW) and were positioned on a heated table while monitoring the basic parameters (temperature, breathing frequency, cardiac frequency, blood pressure and electrocardiogram). The neck region was shaved and the carotid arteries were imaged in transverse view.

Wall thickness was measured in three locations: the lower common carotid artery, the upper common carotid artery (just below the bifurcation), and the internal carotid artery. The measurements were performed on transverse sections at the level where the wall was thickest, including the thickness of atheromatous plaques, when present. We performed the wall measurements so as to include the hyperechoic intimal band and the hypoechoic band belonging to the tunica media.

Color Doppler images were obtained at the level where the walls were thickest for the visualisation of jets and turbulence. Pulsed wave Doppler was used in the regions with high blood velocity (turbulence, jets, when observable, otherwise in the middle of the vessel) to determine the maximum systolic and end-diastolic velocities, as well as systolic acceleration (expressing a stenosis from the inflow, when reduced), resistivity and pulsatility indexes (increasing before a 
stenosis). All data were centralised and comparisons were made between the means, along with statistical tests of significance.

\section{Preparation of carotid artery tissue homoge- nates}

At the end of the ultrasonographic evaluations, the animals were sacrificed (sodium pentobarbital, $60 \mathrm{mg} / \mathrm{kg}$ i.p.). The carotid arteries were removed and were immediately stored at $-80^{\circ} \mathrm{C}$ for further analysis of oxidative stress parameters. Subsequently, the carotid artery was homogenised $(20 \mathrm{mM}$ Tris- $\mathrm{HCl}$ buffer, $\mathrm{pH} 7.4$, in a 1:10 weight/volume ratio) in a Brinkman Polytron Kinematica homogeniser (Lucerne, Switzerland).

\section{Measurement of oxidative stress parameters and antioxidant levels}

Malondialdehyde (MDA) levels were estimated fluorometrically based on the thiobarbituric acid reaction (29) and expressed as nmol/mg protein. Superoxide dismutase (SOD) activity was expressed as U/mg protein Kakkar et al. method) (30).

Reduced glutathione (GSH) and oxidised glutathione (GSSG) were assessed using method Vats et al. (31). They were expressed as nmol/mg pro- tein. The reduced to oxidised glutathione ratio (GSH/GSSG ratio) was assessed.

\section{Statistical analysis}

The results were analyzed using SPSS 17.0 software (Chicago, IL 60606-6412, USA) and were expressed as mean \pm SD. Two-way analysis of variance (ANOVA) followed by Tukey's test for significance was used to compare the effects of quercetin and training. Three-way analysis of variance (ANOVA) was carried out to analyze the cumulative response to quercetin and swimming training in diabetes. A value of $p<0.05$ was considered statistically significant.

\section{Results}

Blood glucose levels and body weights in the STZ-induced experimental model of T1DM

Table 1 shows the blood glucose levels and the body weights (BW) of experimental rats. Animals treated with STZ developed hyperglycemia $(p<0.0001)$ within $96 \mathrm{~h}$, and their blood glucose levels remained higher than those of the control groups (CS, CE, CSQ and CEQ). Training combined with quercetin decreased $(p$ $<0.0001)$ glycemia in diabetic rats. At the end of the study, BW was significantly reduced ( $p$

Table 1. Blood glucose levels and body weights in the STZ-induced experimental model of T1DM

\begin{tabular}{lcccccccc}
\hline & CS & CE & CSQ & CEQ & DS & DE & DSQ & DEQ \\
\hline Initial & 73.0 & 70.9 & 69.7 & 68.8 & 553.1 & 549.8 & 548.5 & 531.0 \\
FBG & \pm 6.99 & \pm 3.21 & \pm 7.71 & \pm 4.84 & $\pm 53.0^{\text {aaa }}$ & $\pm 66.3^{\text {aaa }}$ & $\pm 47.5^{\text {aaa }}$ & $\begin{array}{c} \pm 48.01^{\text {aaa }} \\
(\mathrm{mg} / \mathrm{dL})\end{array}$ \\
\hline Final FBG & 75.9 & 77.7 & 74.8 & 73.4 & 566.5 & 440.0 & 370.1 & 294.3 \\
$(\mathrm{mg} / \mathrm{dL})$ & \pm 4.81 & \pm 4.71 & \pm 3.15 & \pm 3.86 & $\pm 26.8^{\text {aaa }}$ & $\pm 31.2^{\mathrm{b}}$ & $\pm 57.1^{\text {bbb }}$ & $\pm 4.4^{\text {bb }}$ \\
\hline Initial BW & 243.6 & 244.2 & 241.9 & 240.2 & 253.5 & 244.6 & 240.3 & 242.6 \\
$(\mathrm{~g})$ & \pm 8.85 & \pm 10.80 & \pm 10.58 & \pm 18.4 & \pm 14.15 & \pm 16.01 & \pm 11.5 & \pm 7.69 \\
\hline Final BW & 244.7 & 235.9 & 244.1 & 230.6 & 212.3 & 217.7 & 232.7 & 235.6 \\
$(\mathrm{~g})$ & \pm 11.2 & \pm 15.18 & \pm 8.62 & \pm 14.3 & $\pm 8.12^{\mathrm{a}}$ & \pm 10.67 & \pm 15.87 & \pm 13.89 \\
\hline
\end{tabular}

FBG indicates fasting blood glucose and BW indicates body weight. Data are presented as mean \pm SD in the CS (control sedentary rats), CE (control trained rats), CSQ (control sedentary rats that received quercetin), CEQ (control trained rats with quercetin administration), DS (diabetic sedentary rats), DE (diabetic trained rats), DSQ (diabetic sedentary rats that received quercetin), DEQ (trained diabetic rats with quercetin administraion) groups. ${ }^{\text {a }} \mathrm{p}<0.05$ and aaa $\mathrm{p}<0.0001=$ significantly different compared with CS group; ${ }^{\mathrm{b}} \mathrm{p}<0.05$ and ${ }^{\mathrm{bbb}} \mathrm{p}<0.0001=$ significantly different compared with DS group. 
$<0.05)$ in diabetic rats. There were not significant differences $(p>0.05)$ between the final and initial BW in diabetic trained rats that received quercetin.

\section{Vascular US data in the experimental groups}

At the end of the experiment, the common carotid artery (CCA; near the bifurcation) was visualised in all experimental rats using color Doppler and spectral Doppler mode. On US examination, arterial wall plaque formation and wall thickening were more obvious in the DS group than in both the DEQ and DSQ groups (Figure 1 and Table 2). All control groups (CS, CSQ, CE and CEQ) showed normal wall thickness, significantly lower compared to the DS group (Table 2 ). The diabetic trained rats that received quer- cetin (DEQ group) significantly reduced wall thickness $(p<0.005)$.

Among pulsed wave Doppler measurements, pulsatility (PI) and resistivity (RI) indexes showed the most statistically significant increase $(p<0.05)$ in the DS group compared to the control groups (CS, CE, CSQ and CEQ) (Table 2). The diabetic trained rats that received quercetin (DEQ group) restored PI and RI. Significant differences between groups were found for systolic acceleration and peak systolic velocity. Systolic acceleration significantly decreased and peak systolic velocity (PSV) significantly increased $(p<0.05)$ in the DS group compared to the CS group (Table 2). Quercetin administration significantly increased $(p<0.05)$ systolic acceler-
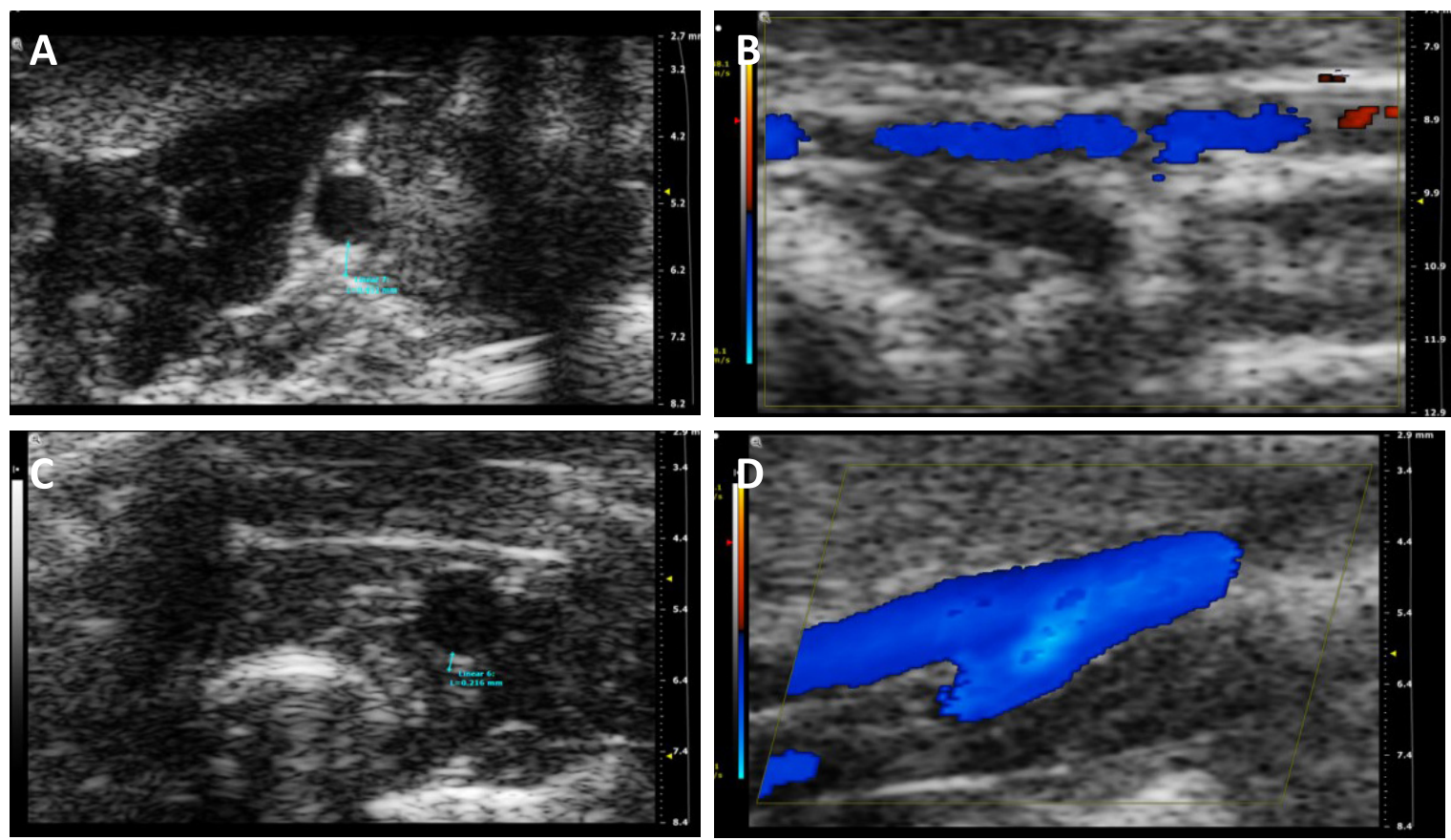

Fig. 1. Representative US parameters of the carotid artery: wall thickness and Doppler measurements in the experimental groups. US changes in the common carotid artery for diabetic sedentary rats

(DS group - A, B) without treatment, respectively after moderate exercise training associated with quercetin administration (DEQ group - C,D). The wall thickness is more increased in the DS group and a plaque is visible for the diabetic sedentary rats (DS group- A, B - transverse view). Doppler studies show signal void at the level of the plaque and increased velocities, with increased PI and RI indexes for the diabetic sedentary rats (DS groupD compared to B). 
Table 2. Representative US parameters of the carotid artery: average wall thickness and Doppler measurements in the experimental groups

\begin{tabular}{lcccccccc}
\hline & CS & CE & CSQ & CEQ & DS & DE & DSQ & DEQ \\
\hline Wall thickness & 0.2 & 0.23 & 0.21 & 0.19 & 0.35 & 0.34 & 0.28 & 0.21 \\
$(\mathrm{~mm})$ & \pm 0.01 & \pm 0.08 & \pm 0.05 & \pm 0.01 & $\pm 0.09 \mathrm{aa}$ & \pm 0.14 & $\pm 0.07 \mathrm{~b}$ & $\pm 0.02 \mathrm{bb}$ \\
\hline Systolic accelera- & 20622 & 22781 & 23778 & 23886 & 14976 & 18486 & 24976 & 25574 \\
tion (mm/s $)$ & \pm 8357 & \pm 15353 & \pm 10741 & \pm 10116 & $\pm 5751 \mathrm{aa}$ & \pm 4224 & $\pm 5751 \mathrm{~b}$ & $\pm 12737 \mathrm{~b}$ \\
\hline Peak systolic ve- & 83.93 & 81.52 & 81.54 & 81.56 & 109.34 & 77.22 & 76.54 & 60.4 \\
locity (PSV) (mm/s) & \pm 28.48 & \pm 5.33 & \pm 4.93 & \pm 47.47 & $\pm 34.38 \mathrm{aa}$ & $\pm 21.83 \mathrm{bb}$ & $\pm 11.78 \mathrm{bb}$ & $\pm 36.08 \mathrm{bb}$ \\
\hline Pulsatility index & 2.76 & 2.84 & 2.55 & 2.95 & 3.18 & 2.82 & 2.56 & 2.86 \\
(PI) & \pm 0.2 & \pm 0.64 & \pm 0.06 & \pm 0.37 & $\pm 0.29 \mathrm{a}$ & $\pm 0.51 \mathrm{~b}$ & $\pm 0.6 \mathrm{~b}$ & $\pm 0.58 \mathrm{~b}$ \\
\hline Resistivity index & 0.86 & 0.84 & 0.83 & 0.75 & 1.14 & 0.85 & 0.85 & 0.88 \\
(RI) & \pm 0.03 & \pm 0.05 & \pm 0.03 & \pm 0.03 & $\pm 0.04 \mathrm{a}$ & $\pm 0.04 \mathrm{~b}$ & $\pm 0.06 \mathrm{~b}$ & $\pm 0.03 \mathrm{~b}$ \\
\hline
\end{tabular}

Data are presented as mean \pm SD in the CS (control sedentary rats), CE (control trained rats), CSQ (control sedentary rats that received quercetin), CEQ (control trained rats with quercetin administration), DS (diabetic sedentary rats), DE (diabetic trained rats), DSQ (diabetic sedentary rats that received quercetin), DEQ (trained diabetic rats with quercetin administraion) groups.a $\mathrm{p}<0.05$ and aa $\mathrm{p}<0.005=$ significantly different compared with CS group; $\mathrm{b} p<0.05$ and $\mathrm{bb} \mathrm{p}<0.005=$ significantly different compared with DS group.

ation and significantly decreased peak systolic velocity in the common carotid artery of diabetic trained rats (DEQ group) compared to control rats (DS group). Oxidative stress parameters and antioxidant levels

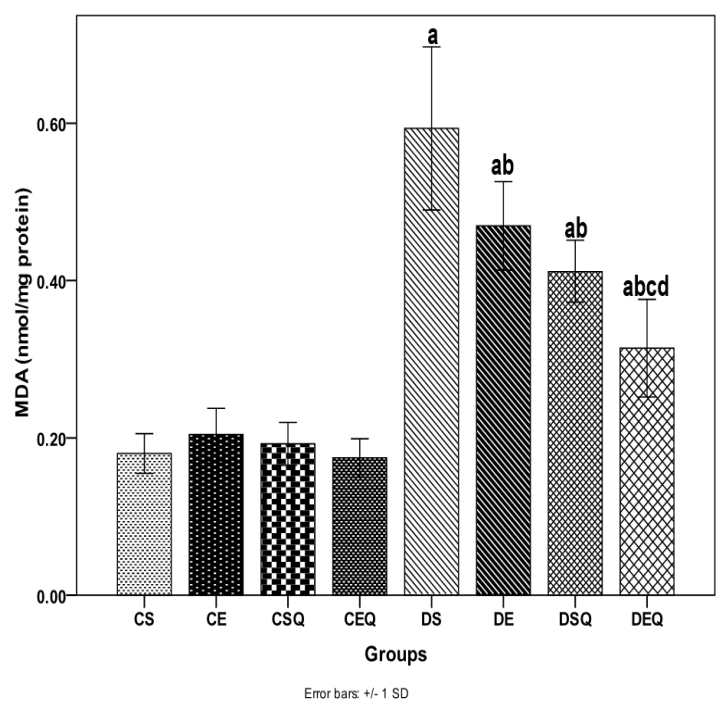

A
This study showed that hyperglycemia induced carotid artery damage, as indicated by a significant increase $(p<0.05)$ in the MDA level of diabetic sedentary rats (Figure $2 \mathrm{~A})$. The results also evidenced a significant decrease $(p<0.05)$

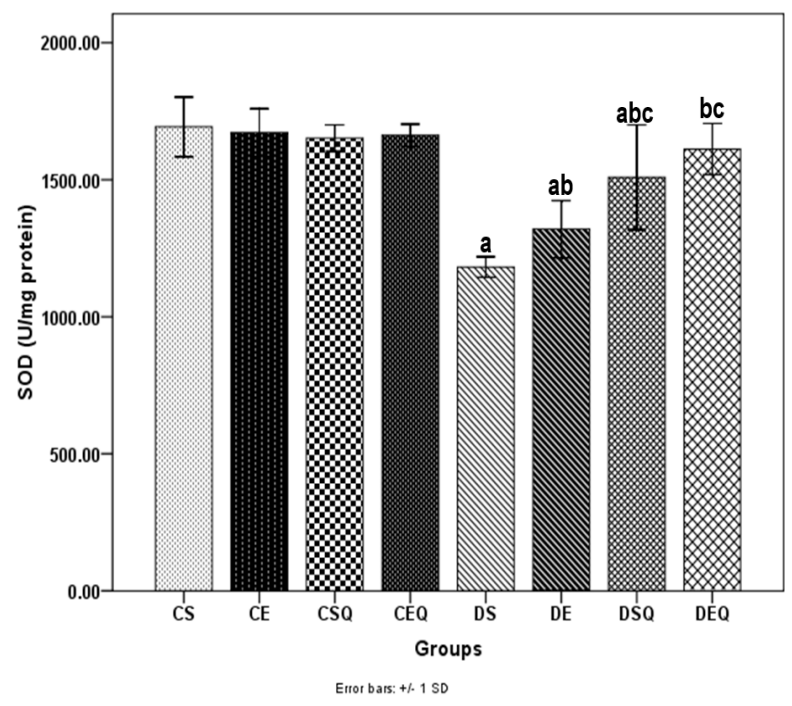

B

Fig. 2. Effect of training combined with quercetin administration on MDA level (nmol/mg protein) (A) and SOD activity (U/mg protein) (B) in the carotid artery of the experimental groups of rats 
in SOD activity (Figure 2B) in the DS group compared to the control groups (CS, CSQ, CE and CEQ).

However, in the diabetic trained rats treated with quercetin $(30 \mathrm{mg} / \mathrm{kg})$, a notable antioxidant effect was observed in the carotid artery (Figure 2). In fact, training combined with quercetin administration in diabetic rats significantly increased $(p<0.05)$ SOD activity and decreased $(p<0.05)$ MDA levels.

Diabetic rats were orally administered with quercetin at the doses mentioned earlier and subjected to moderate exercise training for 5 weeks. Values are given as means $\pm \mathrm{SD}$ in the CS (control sedentary rats), CE (control trained rats), CSQ (control sedentary rats that received quercetin), CEQ (control trained rats with quercetin administration), DS (diabetic sedentary rats), DE (diabetic trained rats), DSQ (diabetic sedentary rats that received quercetin), DEQ (trained diabetic rats with quercetin administraion) groups. ${ }^{\text {a }}$ $p<0.05=$ significantly different compared with CS group; ${ }^{\mathrm{b}} p<0.05=$ significantly different compared with DS group, ${ }^{\mathrm{c}} p<0.05=$ signifi-

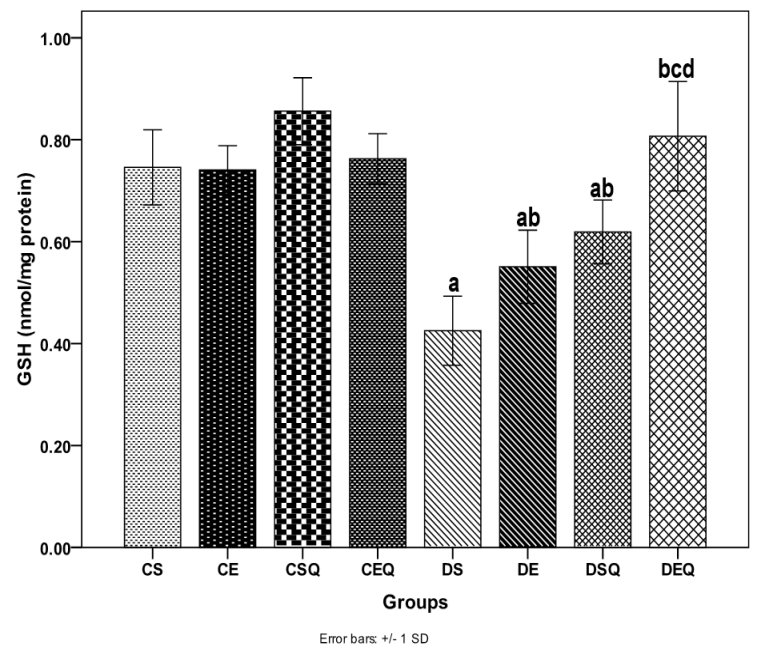

A cantly different compared with DE group and ${ }^{\mathrm{d}}$ $p<0.05=$ significantly different compared with DSQ group.

In Figure 3, the levels of reduced glutathione (GSH) and the GSH/GSSG ratio in the carotid artery homogenates, for the control and diabetic groups are shown. The GSH level (Figure 3A) and the GSH/GSSG ratio (Figure 3B) significantly decreased $(p<0.05)$ in the diabetic carotid artery (DS group) as compared to controls. Both the GSH level and the GSH/GSSG ratio in the diabetic carotid artery were significantly increased $(p<0.05)$ by quercetin administration as compared to controls. Training combined with quercetin treatment significantly increased $(p<$ 0.05 ) the GSH level and the GSH/GSSG ratio in the diabetic carotid artery as compared to untreated diabetic sedentary rats.

Diabetic rats were orally administered with quercetin at the doses mentioned earlier and subjected to moderate exercise training for 5 weeks. Values are given as means $\pm \mathrm{SD}$ in the CS (control sedentary rats), CE (control trained rats), CSQ (control sedentary rats that received quer-

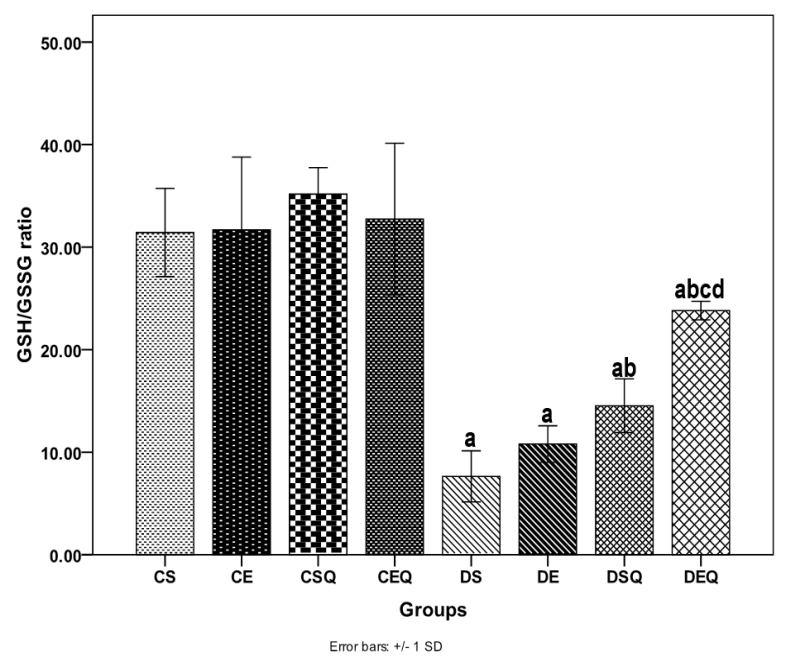

B

Fig. 3. Effect of training combined with quercetin administration on GSH level (nmol/mg protein) (A) and GSH/GSSG ratio (B) in the carotid artery of the experimental groups of rats. 
cetin), CEQ (control trained rats with quercetin administration), DS (diabetic sedentary rats), DE (diabetic trained rats), DSQ (diabetic sedentary rats that received quercetin), DEQ (trained diabetic rats with quercetin administraion) groups. ${ }^{\text {a }}$ $p<0.05=$ significantly different compared with CS group; ${ }^{\mathrm{b}} p<0.05=$ significantly different compared with DS group, ${ }^{\mathrm{c}} p<0.05=$ significantly different compared with DE group and ${ }^{\mathrm{d}}$ $p<0.05=$ significantly different compared with DSQ group.

\section{Discussion}

Quercetin is a natural flavonoid known for its many bioactive properties: anti-inflammatory, antioxidant, hypoglycemic, hypocholesterolemic, etc. In this study, we analysed the potential ameliorative effects of training combined with oral treatment with quercetin on reversing diabetes-induced carotid artery injuries. We performed an ultrasound examination of the carotid artery and carotid oxidative stress parameter evaluations in a streptozotocin (STZ)-induced experimental model of T1DM.

The experimental model of type $1 \mathrm{DM}$ induced by STZ, an antibiotic and chemotherapeutic agent produced by Streptomyces achromogenes, is a reliable experimental model through which diabetes can be obtained in 96 hours and which can be used for longer experimental studies to examine diabetic complications (3,23-28). The limitations of this diabetes animal model are the fact that it can cause post-induction hypoglycemia and that repeated STZ doses can generate pancreatic and liver damage which may result in the death of animals.

Hyperglycemia, hypercholesterolemia and hypertriglyceridemia positively correlate with endothelial dysfunction in T1DM. Inflammation, oxidative stress and hyperglycemia play an important role in the development of atherosclerosis, which leads to diabetic cardiovascular com- plications $(1,6,14,32,35,36)$. The present findings indicate that chronic administration of quercetin to diabetic trained rats, an experimental model of T1DM, significantly decreased FBG levels.

Previous studies show that in diabetes, the combination of training and quercetin treatment restores normal endothelial function and vascular oxidative stress levels through its two major hypoglycemic and antioxidant effects $(3,12,14$ 18,21,32-34). The current study establishes a correlation between the levels of glycemia, oxidative stress and carotid artery wall changes in diabetic rats and evaluates the efficacy of combining quercetin treatment with moderate excercise training in reducing glycemia and oxidative stress and in preventing diabetic vascular complications evidenced in vivo by ultrasound evaluations.

Diabetes causes vascular endothelial dysfunction and hypertrophic remodeling of the carotid artery. The alterations include vessel narrowing, thickening of the tunica media with the deposition of collagen and laminin in the vessel walls, resulting in an accelerate formation of atherosclerosis $(8,35,36)$.

In the present report, we observed both a significant increase in common carotid artery wall thickness (tunica intima + tunica media) and some Doppler parameter changes in STZ-induced diabetic rats. The measurements in our report represent total intima-media thickness and include possible hyperechoic plaques, thus accounting for an increase in size compared to other US studies performed on diabetic rats (37-39).

An increase in the MDA level and a reduction of the GSH level and SOD activity in the carotid artery of diabetic rats were evidenced. The ability of training combined with quercetin administration to protect against free radical-induced damage and the lipid peroxidation process is also shown by the significant decrease in the MDA level and the significantly increased antioxidant 
SOD and GSH levels in the carotid artery of diabetic rats. The changes in the carotid artery wall thickness of diabetic rats were significantly reversed after quercetin treatment, both with and without association of moderate exercise training. In fact, rats with a quercetin regimen and an exercise program had a wall thickness similar to non-diabetic control rats. Quercetin treatment of diabetic trained rats in the present research prevented the development of carotid artery injuries through a diminuation of glycemia and oxidative stress. The carotid artery wall thickness of the diabetic trained_rats treated with quercetin (DEQ) changed insignificantly and atheromatous plaques were absent compared to the control group (CEQ). Ultrasound changes were consistent with the decrease in glycemia and oxidative stress levels in the DEQ group. Quercetin protects the vascular wall from T1DM-induced oxidative injury by increasing the activity of antioxidants, including GSH and SOD, by maintaining mitochondrial function and decreasing the reactive oxygen species level, thus maintaining the cellular redox environment [9,12-15]. Consistently, our data also showed that atheromatous plaques were mostly visible in untreated DM rats and were confirmed on color Doppler images as a signal void in the region of intimal thickening. The diabetic trained rats treated with quercetin (DEQ group) did not show carotid atherosclerotic plaques.

Our results show that fighting some of these effects using quercetin, with its antioxidant, anti-inflammatory, antiatherogenic, hypoglycemic, hypotriglyceridemic and hypocholesterolemic activity in DM $(3,9-12,14,40)$, can provide protection against the development of vascular complications in the carotid arteries.

\section{Conclusions}

Our study demonstrates that oxidative stress and hyperglycemia contribute to the development of vascular damage and atherosclerotic plaques in the carotid artery in a STZ- induced experimental model of T1DM. Moderate exercise training in association with quercetin administration reduces the carotid artery injuries induced by diabetes through a diminution of glycemia and a decrease in oxidative stress levels in the vascular wall. Our results emphasise the possibility of using new combinations to reduce carotid artery alteration in diabetes.

\section{Authors'contribution}

Irina Camelia Chis (Conceptualization; Data curation; Formal analysis; Methodology; Project administration; Software; Writing - original draft; Writing - review \& editing)

Mihai Socaciu (Conceptualization; Investigation; Methodology)

Remus Moldovan (Formal analysis)

Simona Clichici, (Conceptualization; Data curation; Formal analysis; Project administration; Validation)

\section{Conflict of interest}

None to declare.

\section{References}

1. Sena CM, Pereira AM, Seica R. Endothelial dysfunction- A major mediator of diabetic vascular disease. Biochim Biophys Acta. 2013 Dec;1832(12):2216-31. DOI: 10.1016/j.bbadis.2013.08.006

2. Sundaram B, Singhal K, Sandhir R. Anti-atherogenic effect of chromium picolinate in streptozotocin-induced experimental diabetes. J Diabetes. 2013 Mar;5(1):4350. DOI: 10.1111/j.1753-0407.2012.00211.x

3. Chis IC, Muresan A, Adrian O, Andras LN, Simona C. Protective effects of Quercetin and chronic moderate exercise (training) against oxidative stress in the liver tissue of streptozotocin-induced diabetic rats. Physiol Int. 2016 Mar;103(1):49-64. DOI: 10.1556/036.103.2016.1.5

4. Tiwari BK, Pandey KB, Abidi AB, Rizvi SI. Markers of 
Oxidative Stress during Diabetes Mellitus. J Biomark. 2013 Dec; 2013:378790. DOI:10.1155/378790. DOI: $10.1155 / 2013 / 378790$

5. Szabo C. Role of nitrosative stress in the pathogenesis of diabetic vascular dysfunction. Br J Pharmacol. 2009 Mar;156(5):713-27. DOI: 10.1111/j.14765381.2008.00086.x

6. Fowler MJ. Microvascular and Macrovascular Complications of Diabetes. Clin Diabetes 2008 Apr;26(2):7782. DOI: $10.2337 /$ diaclin.26.2.77

7. Umeno A, Horie M, Murotomi K, Nakajima Y, Yoshida Y. Antioxidative and antidiabetic effects of natural polyphenols and isoflavones. Molecules. 2016 May;21(6):708. DOI:10.3390/21060708. DOI: $10.3390 /$ molecules 21060708

8. Wajima D, Nakagawa I, Takamura Y, Aketa S, Yonezawa T, Nakase H. Carotid artery stenosis is exacerbated in spontaneously obese model rats with diabetes. J Atheroscler Thromb. 2014 Jul;21(12):1253-9. DOI: 10.5551/jat.24661

9. Boots AW, Haenen GR, Bast A. Health effects of quercetin: From antioxidant to nutraceutical. Eur J Pharmacol. 2008 May;585(2-3):325-37. DOI: 10.1016/j. ejphar.2008.03.008

10. Pashevin DA, Tumanovska LV, Dosenko VE, Nagibin VS, Gurianova VL, Moibenko AA. Antiatherogenic effect of quercetin is mediated by proteasome inhibition in the aorta and circulating leukocytes. Pharmacol Rep. 2011 Mar;63(4):1009-18. DOI: 10.1016/S17341140(11)70617-X

11. Larson AJ, Symons JD, Jalili T. Therapeutic potential of quercetin to decrease blood pressure: Review of efficacy and mechanisms. Adv Nutr. 2012 Jan;3(1):39-46. DOI: $10.3945 /$ an. 111.001271

12. Jeong SM, Kang MJ, Choi HN, Kim JH, Kim JI. Quercetin ameliorates hyperglycemia and dyslipidemia and improves antioxidant status in type 2 diabetic $\mathrm{db} /$ db mice. Nutr Res Pract. 2012 Jun;6(3):201-7. DOI: 10.4162/nrp.2012.6.3.201

13. Kim JH, Kang MJ, Choi HN, Jeong SM, Lee YM, Kim JI. Quercetin attenuates fasting and postprandial hyperglycemia in animal models of diabetes mellitus. Nutr Res Pract 2011 Apr;52):107-11. DOI: 10.4162/ nrp.2011.5.2.107

14. Chis IC, Coseriu A, Ramona S, Adrian O, Andras LN, Simona C. In vivo effects of Quercetin in association with moderate exercise training in improving strep- tozotocin-induced aortic tissue injuries. Molecules. 2015 DEc;20(12):21770-86. DOI: $10.3390 /$ molecules201219802

15. Chiş IC, Baltaru D, Dumitrovici A, Coseriu A, Radu BC, Moldovan R, Mureşan A. Quercetin ameliorate oxidative/nitrosative stress in the brain of rats exposed to intermittent hypobaric hypoxia. Rev Virtual Quim. 2016 Mar;8(2):369-83. DOI: 10.5935/1984-6835.20160027

16. Chiş IC, Baltaru D, Clichici S, Oniga O, Cojocaru I, Nastasă C. The Effects of a 5-Chromen-yl-thiazolidin-2,4-dione Derivative in Alleviating Oxidative Stress in AdjuvantInduced Arthritis. Rev Chim. 2018 Sep;9(69): 2361-65.

17. Chiş IC, Baltaru D, Dumitrovici A, Coseriu A, Radu BC, Moldovan R, Mureşan A. Protective effects of quercetin from oxidative/nitrosative stress under intermittent hypobaric hypoxia exposure in the rat's heart. Physiol Int. 2018 Sep;105(3):233-46. DOI: 10.1556/2060.105.2018.3.23

18. Pyun SB, Kwon HK, Uhm CS. Effect of exercise on reinnervating soleus muscle after sciatic nerve injury in rats. J Korean Acad Rehabil Med. 1999;23:1063-75.

19. Teixeira de Lemos E, Pinto R, Oliveira J, Garrido P, Sereno J, Mascarenhas-Melo F, et al. Differential Effects of Acute (Extenuating) and Chronic (Training) Exercise on Inflammation and Oxidative Stress Status in an Animal Model of Type 2 Diabetes Mellitus. Mediat Inflamm. 2011 Nov;2011:253061.DOI:10.1155/2011/253061. DOI: $10.1155 / 2011 / 253061$

20. Zhang H, Zhang C. Vasoprotection by dietary supplements and exercise: Role of TNF $\alpha$ signaling. Exp Diabetes Res. 2011 Nov;2012: 972679. DOI:10.1155/2012/972679.DOI: 10.1155/2012/972679

21. Lee S, Park Y Dellsperger KC, Zhang C. Exercise training improves endothelial function via adiponectin-dependent and independent pathways in type 2 diabetic mice. Am J Physiol Heart Circ Physiol. 2011 Aug;301(2):H306-14. DOI: 10.1152/ ajpheart.01306.2010

22. Coskun O, Ocakci A, Bayraktaroglu T, Kanter M. Exercise training prevents and protects streptozotocininduced oxidative stress and beta-cell damage in rat pancreas. Tohoku J Exp Med. 2004 Jul;203(3):145-54. DOI: $10.1620 /$ tjem.203.145

23. Rakieten N, Rakieten ML, Nadkarni MR. Studies on the diabetogenic action of streptozotocin (NSC-37917). Cancer Chemother Rep. 1963 May;29:91-8. 
24. Chang KS, Stevens WC. Endothelium-dependent increase in vascular sensitivity to phenylephrine in long-term streptozotocin diabetic rat aorta. Br J Pharmacol. 1992 Dec;107(4):983-90. DOI: 10.1111/j.14765381.1992.tb13395.x

25. Chis IC, Clichici A, Nagy AL, Oros A, Catoi C, Clichici S. Quercetin in association with moderate exercise training attenuates injuries induced by experimental diabetes in sciatic nerves. Journal of physiology and pharmacology: an official journal of the Polish Physiological. 2017 Dec;68(6):877-86.

26. Szkudelski T. The mechanism of alloxan and streptozotocin action of $\beta$-cells of the rat pancreas. Physiol Res. 2001;50(6):537-46.

27. Oelze M, Knorr M, Schuhmacher S, Heeren T, Otto C, Schulz E, et al. Vascular dysfunction in streptozotocin-induced experimental diabetes strictly depends on insulin deficiency. J Vasc Res. 2011 Jan;48:275-84. DOI: $10.1159 / 000320627$

28. Searls Y, Smirnova IV, Vanhoose L, Fegley B, Loganathan R, Stehno-Bittel L. Time-dependent alterations in rat macrovessels with type 1 diabetes. Exp Diabetes Res. 2012 Jan. DOI:10/1155/2012/278620. DOI: $10.1155 / 2012 / 278620$

29. Conti M, Morand PC, Levillain P. Improved fluoromeric determination of malonaldehyde. Clin Chem. 1991 Jul;37(7):1273-5.

30. Kakkar P, Das B, Viswanthan PN. A modified spectrophotometric assay of superoxide dismutase (SOD). Indian J Biochem Biophys. 1984 Apr;21:130-2.

31. Vats P, Singh VK, Singh SN, Singh SB. Glutathione metabolism under high-altitude stress and effect of antioxidant supplementation. Aviation, Space and Environmental Medicine. 2008 Dec;79(12):1106-11. DOI: 10.3357/ASEM.2305.2008

32. Avogaro A, Albiero M, Menegazzo L, de Kreutzenberg S, Fadini GP. Endothelial dysfunction in diabetes: The role of reparatory mechanisms. Diabetes Care. 2011 May; 34(Suppl 2):S285-90. DOI: 10.2337/dc11-s239

33. Alam MM, Meerza D, Naseem I. Protective effect of quercetin on hyperglycemia, oxidative stress and DNA damage in alloxan induced type 2 diabetic mice. Life Sci. 2014 Jul;109(1):8-14. DOI: 10.1016/j. 1fs. 2014.06.005

34. Rocha RE, Coelho I, Pequito DC, Yamagushi A, Borghetti G, Yamazaki RK, et al. Interval training attenuates the metabolic disturbances in type 1 diabetes rat model. Arq Bras Endocrinol Metab. 2013;57:594-602. DOI: 10.1590/S0004-27302013000800003

35. Akazawa S, Tojikubo M, Nakano Y, Nakamura S, Tamai $\mathrm{H}$, Yonemoto $\mathrm{K}$, et al. Usefulness of carotid plaque (sum and maximum of plaque thickness) in combination with intima-media thickness for the detection of coronary artery disease in asymptomatic patients with diabetes. J Diabetes Investig. 2016 May;7(3):396-403. DOI: $10.1111 /$ jdi.12403

36. Yang J, Fan Z, Yang J, Ding J, Yang C, Chen L. MicroRNA-24 Attenuates Neointimal Hyperplasia in the Diabetic Rat Carotid Artery Injury Model by Inhibiting Wnt4 Signaling Pathway. Int J Mol Sci. 2016 Jun;17(6):E765. DOI: 10.3390/ijms17060765. DOI: 10.3390/ijms 17060765

37. Choi YS, Youn HJ, Youn JS, Park CS, Oh YS, Chung WS. Measurement of the intimal thickness of the carotid artery: comparison between $40 \mathrm{MHz}$ ultrasound and histology in rats. Ultrasound Med Biol. 2009 Jun;35(6):962-6. DOI: 10.1016/j.ultrasmedbio.2008.12.004

38. Wajima D, Nakagawa I, Takamura Y, Aketa S, Yonezawa T, Nakase H. Carotid artery stenosis is exacerbated in spontaneously obese model rats with diabetes. $\mathrm{J}$ Atheroscler Thromb. 2014 Jul;21(12):1253-9. DOI: 10.5551/jat.24661

39. Perez A, Gonzalez-Manzano S, Jimenez R, Perez-Abud R, Haro JM, Osuna A, et al. The flavonoid quercetin induces acute vasodilator effects in healthy volunteers: correlation with beta-glucuronidase activity. Pharmacol Res. 2014 Nov;89:11-8. DOI: 10.1016/j. phrs.2014.07.005

40. Scridon A, Perian M, Marginean A, Fisca C, Vantu A, Ghertescu D, et al. Wistar rats with long-term streptozotocin-induced type 1 diabetes mellitus replicate the most relevant clinical, biochemical, and hematologic features of human diabetes. Rev Romana Med Lab. 2015;23(3):263-74. DOI: 10.1515/rrlm-2015-0028 